\title{
Shoulder injury related to SARS-CoV-2 vaccine administration
}

\author{
Jacky C.K. Chow MD PhD, Sarah L. Koles MD MSc, Aaron J. Bois MD MSc
}

Cite as: CMAJ 2022 January 17;194:E46-9. doi: 10.1503/cmaj.211162

\section{Case 1}

A 62-year-old woman with a history of hypertension, dyslipidemia and neck pain, without a preceding history of shoulder pain, received her first dose of the ChAdOx1 nCoV-19 vaccine. She reported that the needle position felt higher on her arm than previous vaccinations and that she felt pain during the injection. Immediately after the injection, she had restricted range of motion (ROM) of her right shoulder, and active elevation and abduction of her right arm were limited to $45^{\circ}$, making it difficult to perform basic daily activities. Two weeks after vaccine administration, her family physician ordered an ultrasound of the shoulder, which showed mild subacromial bursal distention and a trace of avascular fluid in the anterior subdeltoid bursa, without deltoid muscle fluid collection, or tendon or joint abnormality. After no clinical improvement 1 month after vaccination, the patient privately obtained a magnetic resonance imaging (MRI) study of her right shoulder, which showed focal distention and inflammation in the inferior and lateral aspects of the subdeltoid bursa, consistent with bursitis, at the site where she described receiving her vaccine (Figure 1). There was no alternative cause for her acute pain; the imagining showed acromioclavicular joint osteoarthritis and mild rotator cuff tendinosis that was reasonable for her age.

The patient's family physician managed her symptoms conservatively, without formal physical therapy or corticosteroid injections. Her pain subsided over time. Eight weeks after vaccine administration, her shoulder ROM had improved, and she was able to perform most daily activities. Dull pain persisted at the injection site, which did not require analgesics. At 12 weeks, her symptoms had resolved and she received her second dose of the ChAdOx1 nCoV-19 vaccine without complications.

\section{Case 2}

A 63-year-old man with a history of lower extremity osteoarthritis and paroxysmal atrial fibrillation (not requiring treatment), received his first dose of the ChAdOx1 nCoV-19 vaccine without any immediate complications. Four weeks later, he received his second dose of the same vaccine, which was also uneventful. Two weeks after the second dose, however, he developed progressive pain and swelling to the left upper arm near the injection site, resulting in mild limitation to shoulder ROM. He was still able to perform regular daily

\section{Key points}

- Shoulder injury related to vaccine administration (SIRVA) is an uncommon event, but can occur after vaccination for SARS-CoV-2.

- For patients presenting with unremitting shoulder pain and reduced shoulder range of motion after recently receiving a SARS-CoV-2 vaccine, clinicians should consider SIRVA in their differential diagnosis, along with septic arthritis.

- Patients with SIRVA commonly present with subacromial bursitis, which can be detected using ultrasonography, although magnetic resonance imaging is more sensitive, if available.

- The risk of SIRVA can be reduced by following proper vaccine administration techniques, including using needles of appropriate length for the patient, proper landmarking and avoiding overpenetration in the upper third of the deltoid.

activities. He reported that the vaccine injection site was similar to previous vaccines. Ultrasounds of the left shoulder performed after he presented to the emergency department, showed a complex cystic area within the left deltoid muscle, measuring $31 \times 25 \times 16 \mathrm{~mm}$ (Figure 2). There was no extension to the deep portion of the muscle or subcutaneous tissues, no associated hyperemia to suggest an infectious process and no distention of the subdeltoid bursa. The reporting radiologist identified a trace amount of simple fluid, superficial to the deltoid muscle. These findings were consistent with a subacute intramuscular hematoma, secondary to the recent vaccine administration. The patient's family physician managed his symptoms conservatively with acetaminophen, nonsteroidal antiinflammatory drugs, ice and massage therapy. The pain gradually resolved over the next 4 weeks. At the 7 -week follow-up, the patient continued to report a palpable, albeit smaller, lump in his left upper arm. However, the pain had completely resolved, with no limitations to his shoulder ROM or daily activities.

\section{Discussion}

The National Vaccine Injury Compensation Program in the United States defines shoulder injury related to vaccine administration (SIRVA) as an "adverse event following vaccination thought to be related to the technique of intramuscular percutaneous injection into an arm resulting in trauma from the needle and/or the 

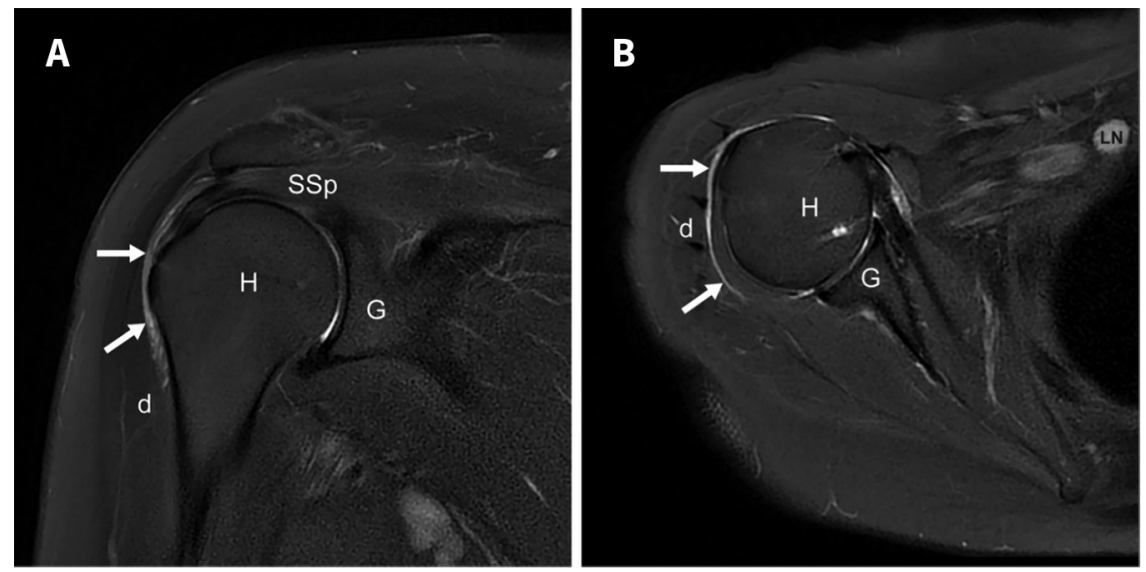

Figure 1: Fat-suppressed magnetic resonance images of a 62-year-old woman with shoulder injury related to vaccine administration, showing subdeltoid bursitis. (A) Coronal and (B) axial views show focal distention and inflammation in the inferior and lateral aspects of the subdeltoid bursa (arrows). The axial view also shows axillary lymphadenopathy, which is likely reactive. Note: $\mathrm{D}=$ deltoid, $\mathrm{G}=$ glenoid, $\mathrm{H}$ = humerus, $\mathrm{LN}=$ lymph node, $\mathrm{SSp}=$ supraspinatus.
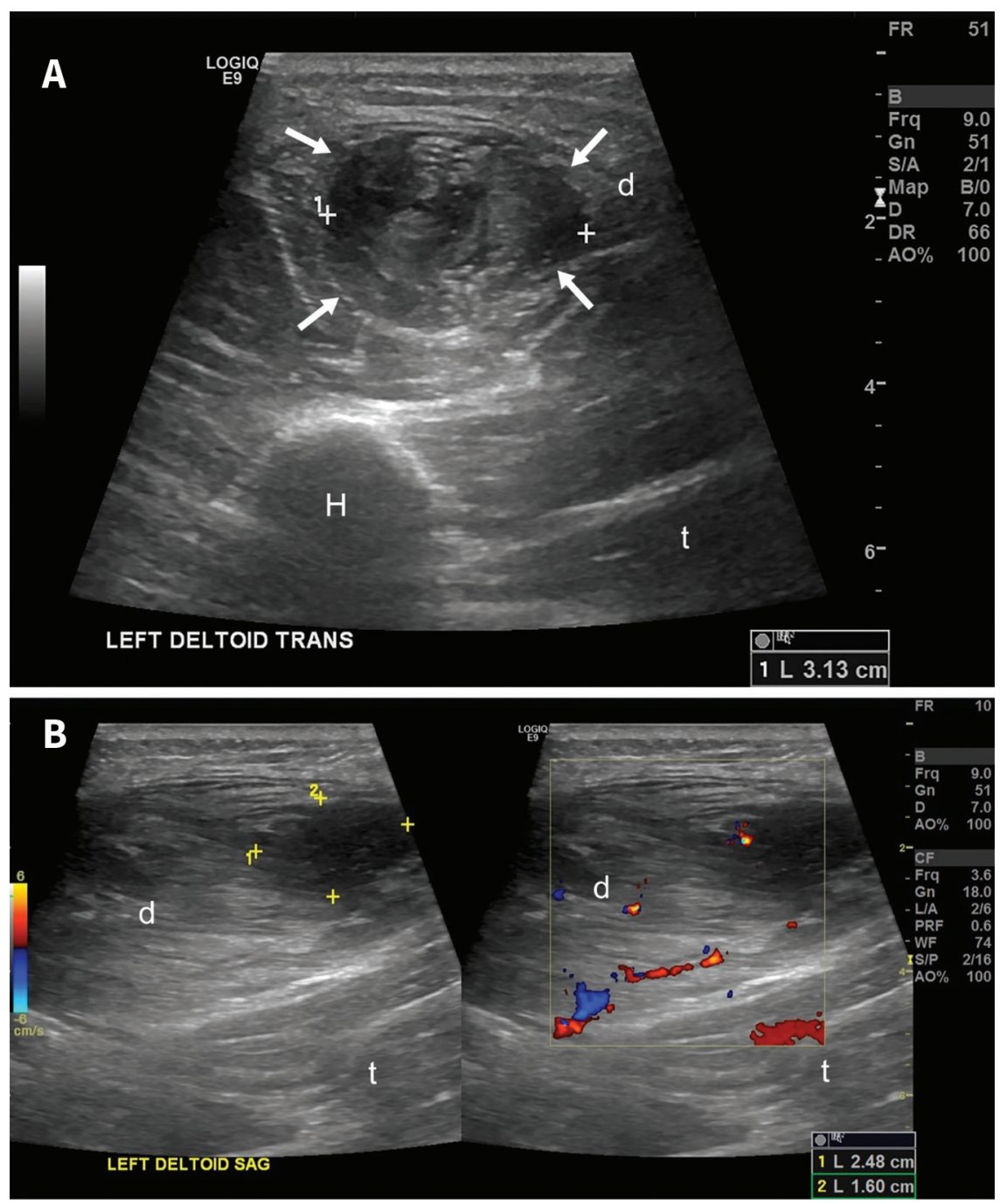

Figure 2: Ultrasounds from a 63-year-old man with injury related to vaccine administration. (A) Transverse and (B) sagittal views show a complex cystic area in the left deltoid muscle (arrows), measuring $31 \times 25 \times 16 \mathrm{~mm}$. Note: $\mathrm{D}=$ deltoid, $\mathrm{H}=$ humerus, $\mathrm{T}=$ triceps. unintentional injection of a vaccine into tissues and structures lying underneath the deltoid muscle of the shoulder." ${ }^{1}$ We present 2 cases of SIRVA after administration of a SARS-CoV-2 vaccine, a patient with subdeltoid bursitis and another with an intramuscular hematoma. Although intramuscular hematoma is a less common manifestation of SIRVA than bursitis, it may result from the needle injuring an intramuscular vessel at the time of vaccination.

\section{Causes and risk factors}

Shoulder injury related to vaccine administration is an uncommon and often preventable iatrogenic event. Epidemiological data are sparse and cases may be underreported. We identified 61 SIRVA cases in a semistructured literature review, and summarized the patients' presenting symptoms, vaccine and clinical course (Appendix 1, available at www.cmaj.ca/lookup/doi /10.1503/cmaj.211162/tab-related-content). Currently, 6 SIRVA cases related to SARSCoV-2 vaccines have been published in addition to these 2 cases, including 1 in Brazil and 1 in Thailand related to the ChAdOx1 $\mathrm{nCoV}-19$ vaccine, a second case in Thailand related to the Sinovac-CoronaVac vaccine and 3 cases in the United States related to mRNA vaccines (mRNA-1273 and BNT162b2) (Appendix 1). Existing evidence does not associate SIRVA with particular vaccines and risk of SIRVA across vaccines is therefore likely equivalent.

The exact cause of SIRVA is unknown, but it may be an immune-mediated reaction to both viral antigens and vaccine adjuvants. $^{2}$ The inflammatory response is not vaccine-specific, but is theorized to be caused by exposing a previously sensitized patient, either naturally or through previous vaccines, to the viral antigen. Similar to the recently reported case in Brazil, the first patient we present in this report had SIRVA after her first dose of the ChAdOx1 nCoV-19 vaccine. We hypothesized that the patient either had exposure to SARS-CoV-2 or to adenovirus, a component of the ChAdOx1 nCoV-19 vaccine (Appendix 1).

Another potential source of shoulder pain related to SIRVA is the presence of pre-existing, asymptomatic shoulder pathology, which becomes provoked by the trauma or injury after vaccine administration. ${ }^{2}$ In our experience, the distention and 
inflammation of the inferolateral subdeltoid bursa occurs because of unintentional vaccine administration at that site and the findings on imaging are distinct from the typical bursal thickening observed with shoulder impingement. This suggests an element of local irritation from the vaccine injection technique, or from inadvertent injection into the bursa rather than the muscle.

Most SIRVA cases reported in the literature occur in women (Appendix 1). This is thought to be related to their body habitus, as women generally have less deltoid muscle mass than men, making them more vulnerable to injuries from longer needles or poor technique. Most patients with confirmed SIRVA reported shoulder pain at the site of injection and that the injection was high in their deltoid muscle. The clinical presentation of the first patient is like other bursitis-related SIRVA cases reported in literature. However, we did not find previous reports of intramuscular hematoma from SIRVA, as with our second patient.

\section{Clinical presentation and diagnosis}

Shoulder injury related to vaccine administration can have a negative impact on a patient's quality of life. Most patients report severe pain within the first 48 hours, and some report delayed symptoms of up to 2 months. ${ }^{3}$ Reduced shoulder ROM is commonly reported, and weakness, altered sensation and fever are less common. ${ }^{2}$ Differential diagnoses of SIRVA include septic arthritis, local inflammation (e.g., bursitis, adhesive capsulitis, tendinitis), nerve damage (i.e., axillary nerve injury), impingement and trauma (i.e., tearing of 1 or more of the rotator cuff tendons). In rare cases, bone erosion, humeral head osteonecrosis, septic arthritis and progressive chondrolysis have been reported..$^{4-7}$

Clinicians should assess for systemic signs of infection, such as fever, joint pain, swelling and erythema. Laboratory investigations that show elevated white cell count and raised inflammatory markers (i.e., C-reactive protein, erythrocyte sedimentation rate or plasma viscosity) may suggest an infective pathology over an inflammatory process. If symptoms of shoulder pain and restricted ROM have not resolved after 1 week from vaccine administration, we suggest obtaining shoulder radiographs to rule out other causes of shoulder pain, and additional soft-tissue imaging to assess for bursitis, namely ultrasonography or MRI. When detecting subacromial bursitis, ultrasonography has a sensitivity ranging from 0.79 to 0.81 , and a specificity ranging from 0.94 to $0.98 .{ }^{8} \mathrm{How}$ ever, ultrasonography is operator-dependent and may underestimate the findings because of direct pressure while scanning; MRI has a higher sensitivity for detecting shoulder pathology. ${ }^{9}$

With the first patient, the bursal thickening was not detected on ultrasonography, but was seen on MRI. The subdeltoid location of bursal thickening is atypical for common subacromial bursitis and can be subtle, which can make detection on ultrasonography challenging. Therefore, MRI can be considered if clinically indicated and if the ultrasonography result is discordant with the clinical presentation.

\section{Treatment and prevention}

For patients with confirmed bursitis on imaging, lifestyle and job modifications, physiotherapy, and nonsteroidal anti-inflammatory drugs are the mainstays of management. Corticosteroid injection can be considered if symptoms are severe and are not improving with conservative measures. ${ }^{2}$ Patients with SIRVA-related subdeltoid bursitis, subacromial bursitis or intramuscular hematoma can be reassured that their shoulder symptoms are likely selflimiting and that they should expect progressive improvement over 4 to 6 weeks. ${ }^{10}$ Surgery is not usually required.

To prevent SIRVA, intramuscular injections to the upper arm should generally avoid overpenetration in the superior one-third of the deltoid muscle, given that the subacromial bursae can have a depth of $0.8-1.6 \mathrm{~cm}$ below the surface of the skin and extend 3-6 cm below the lateral border of the acromion. ${ }^{10}$ Injections administered too superficially may negatively affect the efficacy of the vaccine and increase patient discomfort. ${ }^{11}$ Overpenetration with a needle that is too long for the patient's body habitus or poor administration technique can lead to injury to nearby nerves and vessels (i.e., the anterior branch of the axillary nerve and accompanying posterior humeral circumflex vessels), bursae (e.g., the subacromial and subdeltoid bursae) and tendons (i.e., the rotator cuff), or can potentially lead to a septic process requiring surgical irrigation and débridement (Appendix 1).

In line with current practice and vaccine manufacturers' recommendations, we suggest the patient be seated at the same height as the vaccine administrator, with full visualization of the deltoid, and have their arm resting at their side. ${ }^{2,12}$ The vaccine administrator should select an appropriate needle length by accounting for the patient's age, sex, body mass index and subcutaneous thickness. ${ }^{3}$ The needle should be perpendicular to the skin and should enter 2 to 3 finger widths below the acromion process, avoiding the upper third of the deltoid. ${ }^{12}$ Another method that has been previously suggested to avoid injury to the subacromial or subdeltoid bursa involves placing the patient's hand on their ipsilateral hip (i.e., slightly abducting the shoulder), and landmarking midway between the lateral acromion and deltoid tuberosity when administering the vaccine. ${ }^{13}$

\section{References}

1. National vaccine injury compensation: revisions to the vaccine injury table [80 FR 45132-154]. Fed Reg 2015;80:45132-54. Available: https://www.federalregister. gov/documents/2015/07/29/2015-17503/national-vaccine-injury-compensation -program-revisions-to-the-vaccine-injury-table (accessed 2021 Aug. 1).

2. Atanasoff $S$, Ryan $T$, Lightfoot $R$, et al. Shoulder injury related to vaccine administration (SIRVA). Vaccine 2010;28:8049-52.

3. Martín Arias LH, Sanz Fadrique R, Sáinz Gil M, et al. Risk of bursitis and other injuries and dysfunctions of the shoulder following vaccinations. Vaccine 2017; 35:4870-6.

4. Kuether G, Dietrich B, Smith T, et al. Atraumatic osteonecrosis of the humeral head after influenza A-(H1N1) v-2009 vaccination. Vaccine 2011;29:6830-3.

5. Messerschmitt PJ, Abdul-Karim F, lannotti J, et al. Progressive osteolysis and surface chondrolysis of the proximal humerus following influenza vaccination. Orthopedics 2012;35:e283-6.

6. DeRogatis MJ, Parameswaran L, Lee $P$, et al. Septic shoulder joint after pneumococcal vaccination requiring surgical debridement. HSS J 2018;14:299-301.

7. Erickson BJ, DiCarlo E, Brause B, et al. Lytic lesions in the proximal humerus after a flu shot. JBJS Case Connect 2019;9:e0248.

8. Ottenheijm RP, Bart Staal J, van den Bruel A, et al. Accuracy of diagnostic ultrasound in patients with suspected subacromial disorders: a systematic review and meta-analysis. Arch Phys Med Rehabil 2010;91:1616-25. 
9. Lenza M, Buchbinder R, Takwoingi $Y$, et al. Magnetic resonance imaging, magnetic resonance arthrography and ultrasonography for assessing rotator cuff tears in people with shoulder pain for whom surgery is being considered. Cochrane Database Syst Rev 2013;9:CD009020.

10. Bodor M, Montalvo E. Vaccination-related shoulder dysfunction. Vaccine 2007; 25:585-7.

11. Wiesel BB, Keeling LE. Shoulder injury related to vaccine administration. J Am Acad Orthop Surg 2021;29:732-9.

12. Natanzi N, Hebroni F, Bodor M. Teres minor injury related to vaccine administration. Radiol Case Rep 2020;15:552-5.

13. Behrens RH, Patel V. Avoiding shoulder injury from intramuscular vaccines. Lancet 2021;397:471.

Competing interests: Jacky Chow is co-chair of the student consortium with the International Society of Photogrammetry and Remote Sensing. No other competing interests were declared.

This article has been peer reviewed.

The authors have obtained patient consent.

Affiliations: Department of Radiology (Chow, Koles), Section of Orthopaedic Surgery (Bois), Cumming School of Medicine University of Calgary; McCaig Institute for Bone and Joint Health (Koles, Bois), Calgary, Alta.

Contributors: All authors contributed to the conception and design of the work, drafted the manuscript, revised it critically for important intellectual content, gave final approval of the version to be published and agreed to be accountable for all aspects of the work.

Content licence: This is an Open Access article distributed in accordance with the terms of the Creative Commons Attribution (CC BY-NCND 4.0) licence, which permits use, distribution and reproduction in any medium, provided that the original publication is properly cited, the use is noncommercial (i.e., research or educational use), and no modifications or adaptations are made. See: https://creativecommons.org/ licenses/by-nc-nd/4.0/

Disclaimer: Both cases have been reported to Vigilance Canada.

Correspondence to: Jacky Chow, jckchow@ucalgary.ca

The section Cases presents brief case reports that convey clear, practical lessons. Preference is given to common presentations of important rare conditions, and important unusual presentations of common problems. Articles start with a case presentation (500 words maximum), and a discussion of the underlying condition follows (1000 words maximum). Visual elements (e.g., tables of the differential diagnosis, clinical features or diagnostic approach) are encouraged. Consent from patients for publication of their story is a necessity. See information for authors at www.cmaj.ca. 